\title{
Intermittent pneumatic compression devices combined with anticoagulants for prevention of symptomatic deep vein thrombosis after total knee arthroplasty: a pilot study
}

\author{
This article was published in the following Dove Press journal: \\ Therapeutics and Clinical Risk Management \\ 14 February 2017 \\ Number of times this article has been viewed
}

\section{Pengcheng Liu* \\ Junfeng Liu* \\ Liyang Chen \\ Kuo Xia \\ Xing Wu}

Department of Orthopedics, Shanghai Tenth People's Hospital, School of Medicine, Tongji University, Shanghai, People's Republic of China

*These authors contributed equally to this work
Correspondence: Xing Wu

Department of Orthopedics, Shanghai

Tenth People's Hospital, Number 30I

Middle Yanchang Road, Shanghai 200072,

People's Republic of China

Tel +8613262227613

Email orthopedics_dsyy@I26.com
Objectives: To investigate the effectiveness of intermittent pneumatic compression (IPC) devices combined with anticoagulants for the prevention of deep vein thrombosis (DVT) after total knee arthroplasty (TKA).

Patients and methods: In total 120 patients were involved in this pilot study. Patients in the control group received $10 \mathrm{mg}$ of rivaroxaban per day after surgery. In addition to the prescription of rivaroxaban, IPC devices were used in the experimental group. The diagnosis of DVT was made by compression duplex ultrasound on postoperative day 9 .

Results: The incidence rates of overall, proximal, distal, and intermuscular DVT were $8.3 \%$, $0 \%, 1.67 \%$, and $6.67 \%$ in the experimental group; and $18.3 \%, 0 \%, 5 \%$, and $13.33 \%$ in the control group, respectively. The incidence rates of total, distal, and intermuscular DVT in TKA patients was significantly lower in the experimental group than in the control group. For patients with DVT, enoxaparin was used instead of rivaroxaban, and DVT was found to have disappeared 10-14 days postoperatively.

Conclusion: Compared with the use of rivaroxaban alone, IPC devices combined with anticoagulants can significantly reduce the incidence rate of distal DVT and intermuscular DVT in the early postoperative period after TKA.

Keywords: total knee arthroplasty, deep vein thrombosis, DVT, rivaroxaban, intermittent pneumatic compression

\section{Introduction}

Total knee arthroplasty (TKA), a major lower limb orthopedic surgery, is a significant risk factor for postoperative deep vein thrombosis (DVT). ${ }^{1-3}$ The main danger associated with DVT is that of pulmonary embolism (PE), which can occur when the clot breaks away from the vessel wall and travels as far as the lungs, where it can be fatal. ${ }^{4}$ The increased incidence of osteoarthritis and rheumatoid arthritis in an aging population makes it a particularly worrying concern within the orthopedic community. Combined with the benefit these surgeries provide with regard to both function and quality of life for patients, their prevalence will only continue to increase. DVT is one of the most common in-hospital complications, occurring in $1.4 \%$ of TKA patients. ${ }^{5}$ For patients undergoing TKA, several pharmacological and mechanical therapies are available for the prevention of DVT, including intermittent pneumatic compression (IPC), which are a convenient and effective mechanical prophylaxis measure. ${ }^{1,3}$ 
IPC devices are widely used for DVT prevention and sport recovery. In addition to preventing venous stasis by increasing venous blood flow velocity and cardiac output, studies have also shown that IPC appears to stimulate fibrinolytic activity by inhibiting the tissue factor pathway, suggesting that it affects two of the three criteria of Virchow's triad: reducing stasis, and reversing hypercoagulability by moderating procoagulant activity through elevation of D-dimer. ${ }^{6-10}$ Guidelines from the Chinese Orthopaedic Association recommend the use of IPC for prophylaxis among patients at an intermediate risk for DVT. ${ }^{11}$ Anticoagulation or IPC is recommended for patients at a high risk for DVT, such as those undergoing TKA, while anticoagulation plus IPC is recommended for patients at the highest risk for TKA.,3

Overall, DVT was found to occur in up to $1.4 \%$ of patients enrolled in the Global Orthopaedic Registry. ${ }^{5}$ This would suggest that although many forms of prophylaxis are available, both pharmacological and mechanical, DVT remains an important clinical burden following TKA surgeries. As the risk of bleeding is greatly reduced with mechanical methods of prophylaxis, such as IPC devices, they are attractive options, especially in cases of pharmacological contraindication.

However, the true efficacy of IPC is yet unknown. As such, the objective of this research was to carry out an evaluation on the use of IPC devices along with anticoagulants as DVT prophylaxis methods in orthopedic patients undergoing TKA.

\section{Patients and methods}

Patients aged $>45$ years old, who had osteoarthritis of the knee $(\mathrm{OAK})$ and had undergone unilateral primary TKA surgery, were eligible for inclusion. The exclusion criteria were: inability to give informed consent; history of lower extremity varicose vein; combined organ bleeding risk can not receive drug anticoagulation therapy; anticoagulation treatment (including high dose aspirin); planned follow-up at another hospital; renal failure; heart failure with pitting edema; thrombophlebitis; thromboembolic event during the previous 3 months; other surgery during the previous month; malignancy; hemophilia; and pregnancy. The study protocol and amendments were approved by the Human Research Ethics Committee of Shanghai Tenth People's Hospital, School of Medicine, Tongji University. The study conformed to the standards of the Declaration of Helsinki. All participants provided written informed consent, and verbal and written information to support informed consent was provided to all eligible patients. The methods were carried out in accordance with the approved guidelines from the Human Research Ethics Committee.
Between April 2015 and March 2016, 227 patients with OAK were screened for eligibility at Shanghai Tenth People's Hospital (Figure 1). Of these, 120 patients (56 men and 64 women) were enrolled and assigned to the interventions by an appropriately trained graduate student. Randomization was performed using consecutively numbered sealed envelopes produced by an independent specialist, which were opened after surgery and the application of the means of immobilization. The patients were randomized to anticoagulants rivaroxaban treatment alone (control group) or IPC devices combined with anticoagulants (experimental group).

All patients were operated under the general anesthesia. Patients in the control group received $10 \mathrm{mg}$ of rivaroxaban per day beginning 6-8 hours after the surgery. As well as the prescription of rivaroxaban, IPC devices were used just after the end of the procedure and the true end of anesthetic in the operating theater, and lasted for 48 hours in the experimental group. Patients from the control and the experimental group were allowed to walk in the first 24 hours after surgery.

In the IPC group we applied the VenaFlow ${ }^{\circledR}$ Elite System (DJO, LLC, Vista, CA, USA), which were designed to improve adherence while delivering the same pattern of compression. These IPC devices deliver sequential compression, first around the distal calf, then proximal calf, and then the thigh. Compression is circumferential (around the whole circumference of the leg) and sleeve inflation is gradual. The maximum pressure is $45 \mathrm{mmHg}$. The compression is delivered to one leg at a time at a frequency which depends on the "venous refill time". When the leg is compressed, its volume is reduced. Between compressions the veins refill and the volume of the leg increases. The changes in volume are detected by the IPC controller, which detects movement of air between the sleeves and the controller. The frequency of compression is higher if venous refill is faster, such as when the legs are dependent. This system aims to maximize venous flow.

Preoperative baseline such as whether patients had diabetes, high blood pressure and other medical complications were recorded. In total, 60 patients were randomized to the experimental group and 60 to the control group at the intention-to-treat stage (Figure 1). Each group comprised 28 men and 32 women, at final analysis. Variables including age, gender, body mass index (BMI), smoking status, time to surgery, duration of surgery, and the daily duration of IPC were recorded for each patient.

The diagnosis of DVT in the lower extremities was made by compression duplex ultrasound (CDU). A technician blinded to patients' allocation performed CDU of both legs on postoperative day 9. The incidence rate of DVT and 


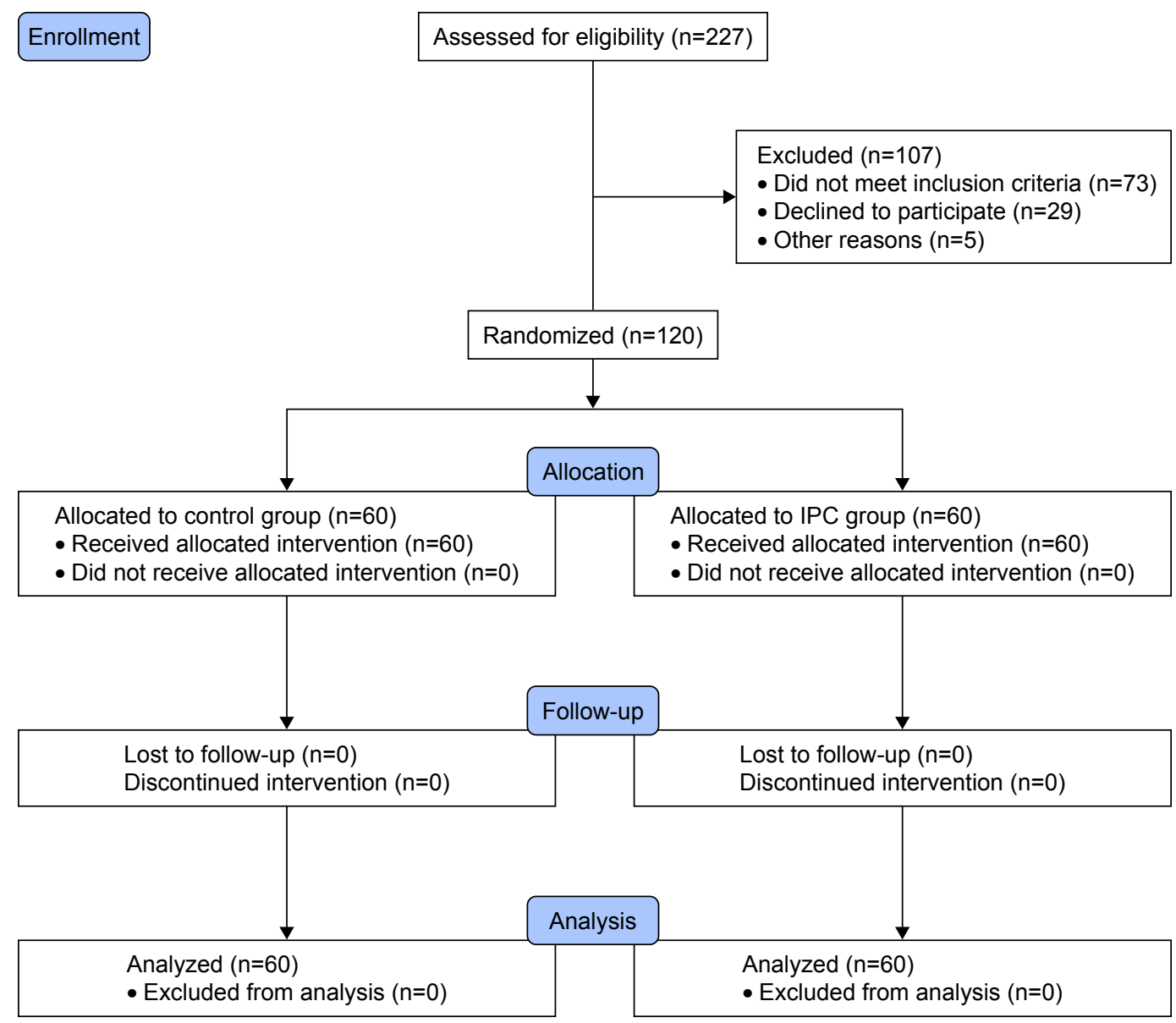

Figure I The flow chart of participants randomization.

Abbreviation: IPC, intermittent pneumatic compression.

symptomatic PE was recorded. The primary outcome was DVT in popliteal or femoral veins, detected on a screening CDU, or any symptomatic DVT in the proximal veins, confirmed by imaging, within 21 days of randomization. The incidence rates of total DVT, proximal DVT (p-DVT; proximal to the trifurcation of the popliteal vein), distal DVT (d-DVT; in the anterior tibial vein, posterior tibial vein, or peroneal vein) and intermuscular DVT were recorded. Computed tomography pulmonary angiography was used to confirm PE if it was suspected. Secondary outcomes included death, any DVTs, symptomatic DVTs, PE, skin breaks on the legs, falls with injury or fractures, and duration of IPC devices use occurring within 21 days of randomization, symptomatic venous thromboembolism, mean intraoperative blood loss, and length of initial hospital stay measured 1 month after randomization.

\section{Statistical analysis}

All variables were summarized with standard descriptive statistics including mean, standard deviation, and frequency. Categorical variables were compared with Pearson's chi-squared test, or Fisher's exact test if any cell had an expected cell count less than 5. Continuous variables, such as age and time to surgery, were analyzed for skewed distributions or outliers, of which none was found. Therefore, differences between groups were analyzed using the Student's $t$-test for independent samples. All statistics, descriptive as well as analytic, were calculated using SPSS (v22.0) software (SPSS Inc., Chicago, IL, USA). The level of significance was set to $\leq 0.05$ (two-tailed).

\section{Results}

Patients had a mean age of 64 years (49-87). There were no significant differences in the distribution of age, gender, smoking status, BMI, diabetes, high blood pressure, time to surgery or duration surgery between groups. Experimental group patients underwent a mean of 66 hours (49-78) of selfrecorded IPC. This correlated well with the device-recorded usage of a mean of 67 hours (50-82).

We allocated 60 patients to the experimental group and 60 to the control group. The primary outcome incidence rates of total DVT occurred in five $(8.3 \%)$ of 60 patients 
allocated to the experimental group and 11 (18.3\%) of 60 patients allocated to the control group $(P<0.01)$, giving a relative risk reduction of $10 \%$. The incidence rates of DVT from specific location in IPC compared with no-IPC patients was p-DVT in $0(0 \%)$ versus $0(0 \%)(P=1.0)$, d-DVT in one $(1.67 \%)$ versus three $(5 \%)(P<0.001)$ and intermuscular DVT in four $(6.67 \%)$ versus eight $(13.33 \%)(P<0.001)$. No deaths or symptomatic PE occurred in either group. Secondary outcome in IPC compared with no-IPC patients was skin breaks in three (5\%) versus two $(3.33 \%)(P=0.062)$, mean intraoperative blood loss $(322.5 \pm 167.38 \mathrm{~mL})$ versus (336.2 $\pm 189.15 \mathrm{~mL})(P=0.524)$.

A total of 16 patients were confirmed for the diagnosis of DVT by CDU in both groups. These 16 patients discontinued IPC devices immediately and changed the anticoagulant drugs rivaroxaban to enoxaparin by using therapeutic dose of 100 anti-Xa IU/kg. The patients with p-DVT and d-DVT were asked to lie in bed and not move their lower limbs, while the patients with intermuscular DVT were allowed to get out of bed with normal rehabilitation exercises. These patients were reviewed for lower extremity venous by CDU before discharge from the hospital (10-14 days postoperatively), all blood clots had dissolved away.

\section{Discussion}

The importance of DVT prophylaxis arises from the increased risk of developing a fatal PE in TKA patients. DVT is thought to affect $1 \%-2 \%$ of all hospitalized patients. ${ }^{12}$ Even though most DVTs start and remain confined to the calf muscles, with the majority resolving spontaneously, the risk of proximal propagation and development of PE increases according to the severity of the initiating prothrombotic stimulus. ${ }^{13}$ Clinical diagnosis of $\mathrm{PE}$ is often challenging, and a certain proportion of patients are diagnosed with PE only at postmortem examination. ${ }^{14}$ Therefore, prevention of DVT and subsequent $\mathrm{PE}$ is the first priority to reduce the perioperative mortality after TKA.

Correlation analysis demonstrated that patients aged $>39$ years exhibited an almost fivefold increased risk of DVT. Other risk factors, such as BMI, smoking status, time to surgery, and duration of surgery did not significantly affect the risk of DVT. This single risk factor of age should be borne in mind when considering a patient's thromboprophylaxis. Also, the risk of developing DVT can be different depending on race and environmental factors (eg, eating habits, lifestyle, and obesity). ${ }^{1}$

Stannard et $\mathrm{al}^{15}$ looked at the difference between IPC of the foot combined with enoxaparin (group A) against enoxaparin alone (group B) in the prevention of DVT following trauma. In group A, 9/103 patients developed DVT compared with 13/97 in group B; this difference was not found to be statistically significant. However, 2/103 in group A and $11 / 97$ in group B developed large $(>2 \mathrm{~cm})$ or occlusive DVT $(P=0.025)$. Furthermore, two patients in group B were diagnosed with PE while none occurred in group A. Those who developed DVT required more blood transfusions (mean, 7.4 units) compare with others (mean, 3.9).

One interesting question is whether the cuff length (foot, calf, thigh, or combined) influences the risk of DVT. However, in orthopedic patients, there was no difference in efficacy in a study examining thigh versus calf-length devices ${ }^{16}$ as well as in a study examining plantar versus leg-length devices. ${ }^{17}$ A Cochrane meta-analysis examined the use of IPC devices with different length of garment after hip surgery ${ }^{18}$ and found only one eligible study reporting no DVT events.

In orthopedic surgery, IPC devices can be placed on the nonsurgical leg at induction and on both legs after wound closure; ${ }^{19}$ the American College of Chest Physicians Evidence-Based Clinical Practice Guidelines recommend continuing the devices for 10-14 days after surgery. ${ }^{20}$ These guidelines also suggest using IPC devices during the hospital stay in addition to pharmacologic prophylaxis. For patients at high risk of DVT, the length of antithrombotic prophylaxis is recommended to be extended to 35 days after surgery, but the role of IPC devices for 35 days, either alone or in combination with a pharmacologic agent, remains to be determined.

Most recommendations suggest that IPC devices play a role as an additional modality that can provide additional efficacy to prevent DVT when it is used in combination with pharmacologic therapy in the highest risk patients. ${ }^{21}$ In all patients, a systematic risk assessment is a critical prerequisite to the choice of any thromboprophylactic agent. Risk stratification is frequently assessed by the Padua Prediction Score for major orthopedic surgery patients with restricted mobility are usually considered high risk. Further studies are needed to assess practical clinical questions that remain unanswered, including optimal cuff length and location, sequence and duration of pressure, and whether use of IPC devices in an outpatient setting can be effective and achieve good compliance.

Due to the low incidence rate of DVT, having an insufficient sample size is an inherent limitation of such studies. There are several limitations in this study. Apart from the inherent limitation of a small sample size, we used a surrogate indicator, which was asymptomatic DVT. 
Furthermore, we did not demonstrate the safety of either of the thromboprophylaxes, as the study was terminated before a sufficient number of patients were enrolled.

Nonetheless, our study suggested that IPC devices may have contributed to lowering the risk of DVT among Chinese surgical patients with TKA. IPC devices combined with anticoagulants can significantly reduce the incidence rate of d-DVT and intermuscular DVT in the early postoperative period after TKA. A combination of both IPC devices and chemoprophylaxis appears to be the best approach for prevention of DVT in TKA patients. Our finding suggests adding the pneumatic compression therapy to the conventional anticoagulation prophylaxis in TKA patients. Further studies are required to confirm the findings of this preliminary analysis.

\section{Acknowledgment}

This project was supported by the National Science Foundation of China (grant number 81371950).

\section{Disclosure}

The authors report no conflicts of interest in this work.

\section{References}

1. JCS Joint Working Group. Guidelines for the diagnosis, treatment and prevention of pulmonary thromboembolism and deep vein thrombosis (JCS 2009). Circ J. 2011;75(5):1258-1281.

2. Anderson FJ, Spencer FA. Risk factors for venous thromboembolism. Circulation. 2003;107,23(1):I9-I16.

3. Kreutzer L, Minami C, Yang A. Preventing venous thromboembolism after surgery. JAMA. 2016;315(19):2136.

4. Epley D. Pulmonary emboli risk reduction. J Vasc Nurs. 2000;18(2): 61-70.

5. Cushner F, Agnelli G, FitzGerald G, Warwick D. Complications and functional outcomes after total hip arthroplasty and total knee arthroplasty: Results from the Global Orthopaedic Registry (GLORY). Am J Orthop (Belle Mead NJ). 2010;39(9):22-28.

6. Tarnay TJ, Rohr PR, Davidson AG, et al. Pneumatic calf compression, fibrinolysis, and the prevention of deep venous thrombosis. Surgery. 1980;88(4):489-496.

7. Salzman EW, McManama GP, Shapiro AH, et al. Effect of optimization of hemodynamics on fibrinolytic activity and antithrombotic efficacy of external pneumatic calf compression. Ann Surg. 1987;206(5): 636-641.
8. Jacobs DG, Piotrowski JJ, Hoppensteadt DA, Salvator AE, Fareed J. Hemodynamic and fibrinolytic consequences of intermittent pneumatic compression: Preliminary results. J Trauma. 1996;40(5): 710-717.

9. Comerota AJ, Chouhan V, Harada RN, et al. The fibrinolytic effects of intermittent pneumatic compression: Mechanism of enhanced fibrinolysis. Ann Surg. 1997;226(3):306-314.

10. Chouhan VD, Comerota AJ, Sun L, Harada R, Guaguhan JP, Rao AK. Inhibition of tissue factor pathway during intermittent pneumatic compression: A possible mechanism for antithrombotic effect. Arterioscler Thromb Vasc Biol. 1999;19(11):2812-2817.

11. Chinese Orthopaedic Association. [Prevention of venous thromboembolism after major orthopaedic surgery]. Chinese Journal of Orthopaedics. 2007;27:790-792. Chinese.

12. Line BR. Pathophysiology and diagnosis of deep venous thrombosis. Semin Nucl Med. 2001;31(2):90-101.

13. Kearon C. Natural history of venous thromboembolism. Circulation. 2003;107(23 Suppl 1):I22-I30.

14. Goldhaber SZ, Hennekens CH, Evans DA, Newton EC, Godleski JJ. Factors associated with correct antemortem diagnosis of major pulmonary embolism. Am J Med. 1982;73(6):822-826.

15. Stannard JP, Lopez-Ben RR, Volgas DA, et al. Prophylaxis against deep-vein thrombosis following trauma: A prospective, randomized comparison of mechanical and pharmacologic prophylaxis. J Bone Joint Surg Am. 2006;88(2):261-266.

16. Soderdahl DW, Henderson SR, Hansberry KL. A comparison of intermittent pneumatic compression of the calf and whole leg in preventing deep venous thrombosis in urological surgery. J Urol. 1997; 157(5):1774-1776.

17. Anglen JO, Bagby C, George R. A randomized comparison of sequentialgradient calf compression with intermittent plantar compression for prevention of venous thrombosis in orthopedic trauma patients: preliminary results. Am J Orthop (Belle Mead NJ). 1998;27(1):53-58.

18. Zhao JM, He ML, Xiao ZM, Li TS, Jiang H. Different types of intermittent pneumatic compression devices for preventing venous thromboembolism in patients after total hip replacement. Cochrane Database Syst Rev. 2014(12):D9543.

19. Levy YD, Hardwick ME, Copp SN, Rosen AS, Colwell CJ. Thrombosis incidence in unilateral vs Simultaneous bilateral total knee arthroplasty with compression device prophylaxis. J Arthroplasty. 2013; 28(3):474-478.

20. Falck-Ytter Y, Francis CW, Johanson NA, et al. Prevention of VTE in orthopedic surgery patients: Antithrombotic Therapy and Prevention of Thrombosis, 9th ed: American College of Chest Physicians Evidence-Based Clinical Practice Guidelines. Chest. 2012;141(2): e278S-e325S.

21. Sadaghianloo N, Dardik A. The efficacy of intermittent pneumatic compression in the prevention of lower extremity deep venous thrombosis. J Vasc Surg Venous Lymphat Disord. 2016;4(2):248-256.
Therapeutics and Clinical Risk Management

\section{Publish your work in this journal}

Therapeutics and Clinical Risk Management is an international, peerreviewed journal of clinical therapeutics and risk management, focusing on concise rapid reporting of clinical studies in all therapeutic areas, outcomes, safety, and programs for the effective, safe, and sustained use of medicines. This journal is indexed on PubMed Central, CAS,

\section{Dovepress}

EMBase, Scopus and the Elsevier Bibliographic databases. The manuscript management system is completely online and includes a very quick and fair peer-review system, which is all easy to use. Visit http://www.dovepress.com/testimonials.php to read real quotes from published authors. 